\title{
Microscopical studies of necrotising scleritis. II. Collagen degradation in the scleral stroma
}

\author{
ROBERT D. YOUNG ${ }^{1}$ AND PETER G. WATSON ${ }^{2}$ \\ From 'Strangeways Research Laboratory, Cambridge, and the ${ }^{2}$ Department of Ophthalmology, \\ Addenbrooke's Hospital, Cambridge
}

SUMMARY Electron microscopy of tissue excised during surgery in eight cases of advanced anterior necrotising scleritis showed degradation of collagen in the scleral stroma by both intracellular and extracellular mechanisms. In the first of these mechanisms cells resembling active fibroblasts and macrophages were observed in the process of phagocytosis of collagen fibrils into vacuoles associated with dense cytoplasmic granules. In the second mechanism collagen fibrils in large areas of the scleral stroma appeared swollen and unravelled, or completely solubilised, without close association with stromal cells. Both activation and degeneration of stromal fibrocytes were evident in zones of extracellular fibril degradation. These changes appeared to take place prior to invasion of the stroma by cells of the granuloma.

As with most other connective tissues collagen is the major structural component of the scleral matrix. The tough yet resilient properties of the sclera are determined largely by the structure and organisation of collagen fibrils within the scleral lamellae which comprise the eyeball.

Collagen is inherently stable both chemically and physically and becomes increasingly resistant to degradation with age owing to the formation of additional crosslinks within the fibril. Little conclusive evidence is available concerning the precise mechanisms of collagen turnover in vivo, either in the slow controlled turnover characteristic of healthy tissues or in diseased tissues showing rapid degradation of collagen. In spite of this a wide range of proteolytic enzymes, some operating with acid, others with neutral $\mathrm{pH}$ optima, are known to be capable of varying degrees of collagen degradation, at least in vitro. From this has arisen the generally accepted view that in vivo there may be two mechanisms of collagen degradation, one taking place inside the cell and involving acid proteases within the lysosomes, the other involving neutral proteases released into the connective tissue matrix. ${ }^{1}$

Previous histological studies of the scleral matrix during necrotising scleritis have made only brief reference to the nature of stromal changes associated

Correspondence to $\operatorname{Dr}$ R. D. Young, Strangeways Research Laboratory, Worts' Causeway, Cambridge CB1 4RN. with the granuloma. Hyalinisation, ${ }^{2}$ or splaying and fraying of collagen fibres in the presence of areas of 'smudgy' necrosis, ${ }^{3}$ have been described. In a review of ocular involvement in collagen diseases François ${ }^{4}$ reported destruction of collagen by so-called fibrinoid degeneration and necrosis in necrotising scleritis. Loss of structure of the scleral lamellae was marked by accumulation of homogeneous, eosinophilic material considered to be a mixture of fibrin and collagen degradation products.

No previous ultrastructural studies of collagen changes in necrotising scleral disease have been reported. In this study electron microscopy was used to determine the nature of scleral destruction in tissue from eight cases of necrotising scleritis. Evidence was obtained for two mechanisms of collagen degradation in this disease.

\section{Observations}

In normal scleral stroma, collagen fibres branch and interweave extensively and vary in both size and shape. Each fibre is composed of many parallel collagen fibrils which appear to be fusiform in shape. Fibril size varies considerably, with the largest and more heterogeneous fibril diameters found in the outer stroma.

Electron microscopy revealed two mechanisms of collagen fibril degradation in the scleral stroma of these patients. In three patients, all of whom 


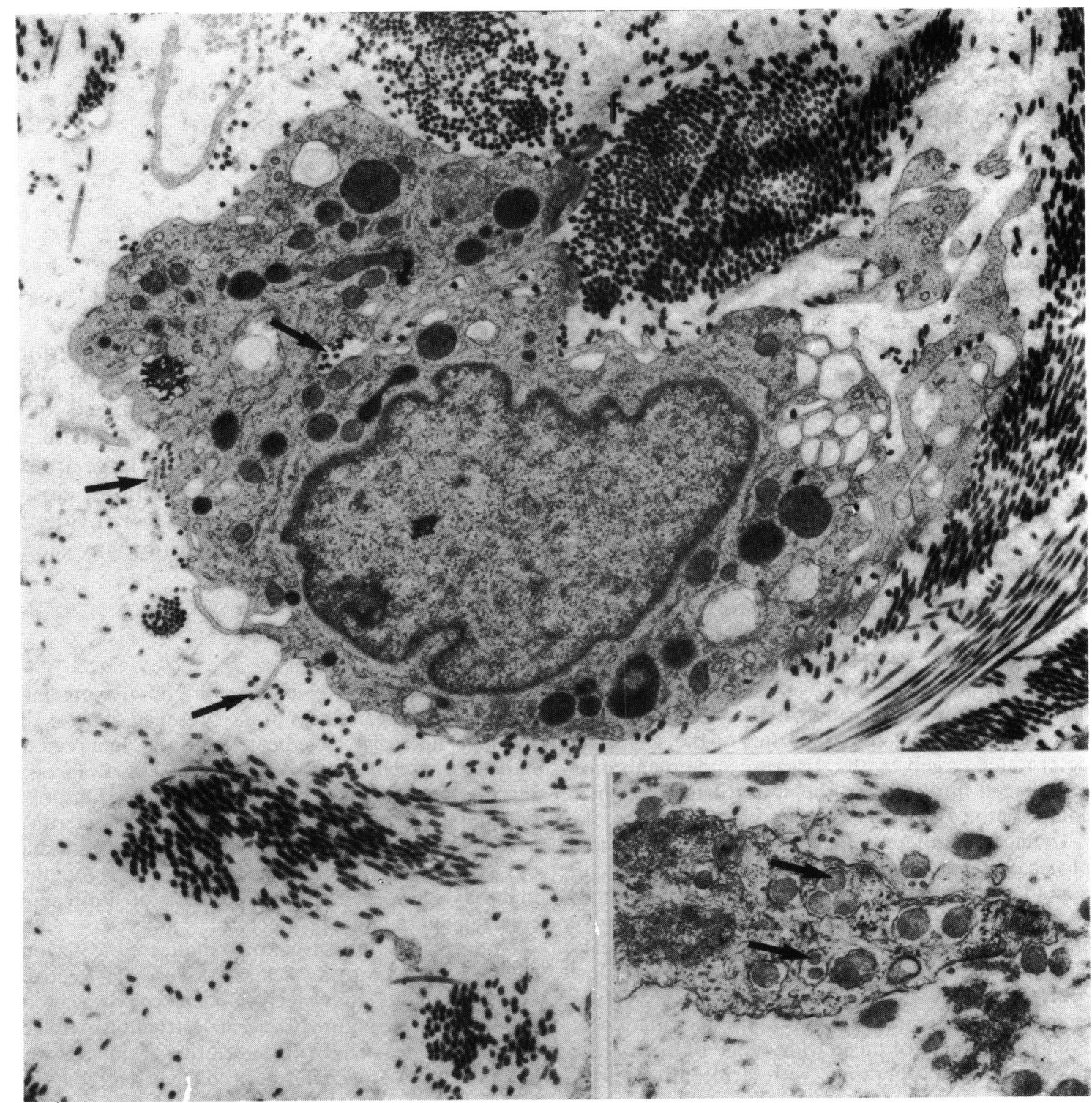

Fig. 1 Electron micrograph of scleral stroma in case 8 showing a rounded cell containing numerous dense cytoplasmic granules. The cell is attached to a collagen fibre seen in transverse section (f) near top centre. Individual or small groups of collagen fibrils are located close to the cell membrane, pseudopodia, and in vacuoles within the cytoplasm (arrows). $(\times 10500)$. Inset: Electron micrograph of a pseudopodium of a phagocytic scleral cell with collagen fibrils of variable diameters (arrows) in separate cytoplasmic vacuoles. $(\times 25000)$.

developed scleritis after ocular surgery, close associations and interaction between cells and collagen fibrils in the stroma was observed at the centre of the ulcer. Scleral collagen fibres were widely separated by diffuse tracts of homogeneous material occupied by active stromal cells. Small groups of individual collagen fibrils were scattered between compact fibres. Fibrils or fibril fragments were often found singly or in small groups in close apposition to the plasma membranes of the stromal cells (Fig. 1). Fibrils enclosed in invaginations of the cell membrane or in membrane-bound vacuoles within the cytoplasm were numerous in most cells. Several fibrils of variable diameter were often seen in any one phagocytic cell 


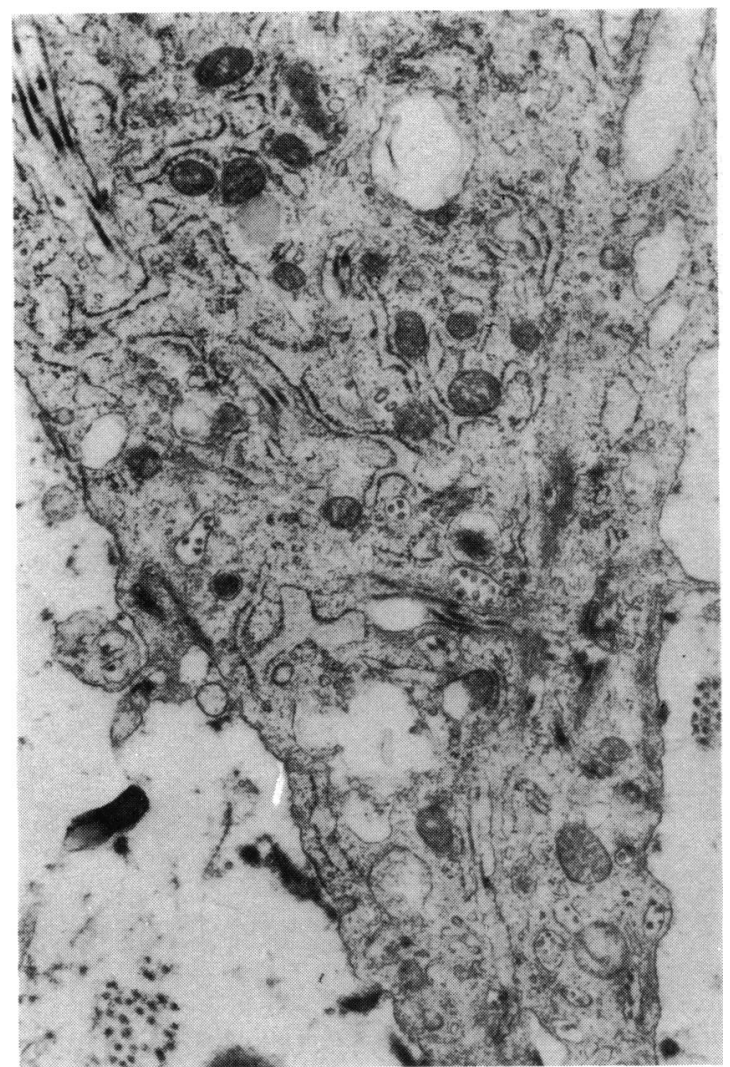

Fig. 2 Electron micrograph of a phagocytic scleral cell from case 7 showing collagen fibrils in longitudinal and transverse aspects, in separate membrane-bound vacuoles. $(\times 16500)$.

but only rarely within the same vacuole (Fig. 1 inset). Similarly, intracellular fibrils in both longitudinal and transverse orientation were often observed in one cell but within separate phagosomes (Fig. 2).

Cells involved in phagocytosis of collagen were flattened and attenuated or rounded in shape (Figs. $1,2,3)$. Nuclei were elongate and slender or rounded with indentations, with fine peripheral heterochromatin. Organelles consisted primarily of numerous mitochondria, with rough-surfaced endoplasmic reticulum and Golgi membranes normally evident. In the more rounded cells many electrondense, membrane-bound granules were typically present in the cytoplasm, polarised towards zones of prominent pseudopod formation and collagen phagocytosis (Fig. 1). Secondary phagosomes were also present. All phagocytic cells were characterised by highly active cell surfaces. Slender filopodia, often filled with fibril-containing vacuoles, extended like tentacles into the surrounding matrix. No extracellular degranulation was seen at sites of fibril

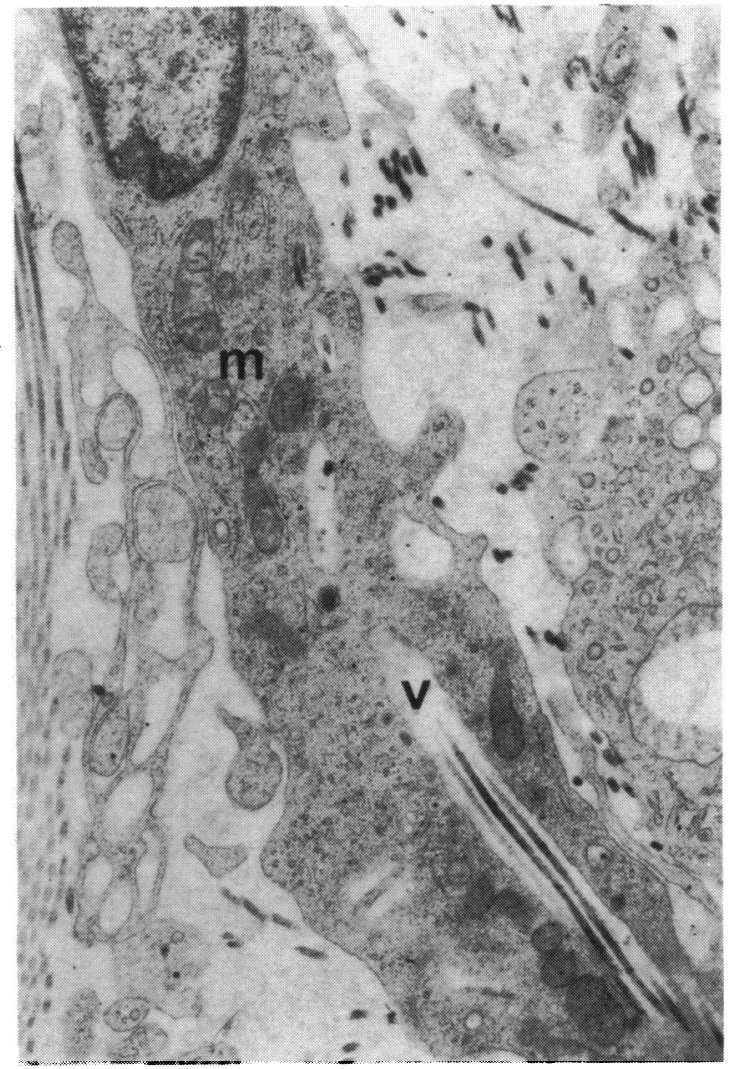

Fig. 3 Electron micrograph of scleral stroma from case 8. An elongate scleral cell with prominent mitochondria (m), but no cytoplasmic granules, contains collagen fibrils within an obliquely sectioned vacuole $(\mathrm{v}) .(\times 14000)$.

engulfment. However, mast cells with partially discharged granules were frequently observed at these sites.

In case 4 the centre of the lesion was marked by almost total destruction of collagen fibrils. Polymorphonuclear leucocytes were relatively numerous (Fig. 4) in addition to mast cells and cells similar to those described above. Pericellularly a diffuse flocculent suspension contained fibrin and small groups of intact collagen fibrils and was bordered by lymphocytes and plasma cells of the granuloma.

Traversing the zone of scleral inflammation, from the ulcer centre, described above, across surrounding granulomatous tissue into peripheral regions of the sclera, the sclera appeared by light microscopy to be undamaged. However, electron microscopy revealed that matrix resorption, as indicated by changes in collagen fibril structure, was already in progress in the absence of inflammatory cells (Fig. 5).

In four cases collagen fibres which appeared initially 




Fig. 4 Accumulation of neutrophils at the centre of scleral ulcer in case 4, where collagen destruction is total. $(\times 9000)$.

to be intact were found on closer examination of individual fibrils to be in the early stages of fibril breakdown. This appeared in longitudinal section as a series of clefts running spirally or diagonally along the fibril (Fig. 6). In transverse section affected fibrils had a serrated or indented profile, distinguishing them clearly from the smooth contours of their undamaged neighbours (Fig. 6 inset). Fibrils showed increased interfibrillar distance in comparison with normal tissue. No perifibrillar coating or precipitate was present, though a diffuse interfibrillar staining was normally observed. A further change was illustrated by fibrils with longitudinal clefts, which appeared expanded or swollen at discrete foci along their length (Fig. 7). Fine microfibrils were visible at these points, which displayed a faint $31 \mathrm{~nm}$ periodicity in comparison with the $45 \mathrm{~nm}$ periodicity of the intact regions of the fibril. In transverse section juxtaposed fibrils varied widely in the extent of their swelling. Some showed a two to four times increase in diameter over apparently unaffected fibrils. Extensive swelling of the majority of fibrils was present in some collagen fibres sectioned longitudinally (Fig. 8). Elsewhere affected fibrils undulated in and out of the section plane, making impossible an assessment of the extent of fibril damage. In some fibres skeins of parallel intact fibrils with regular diameters and crossbanding underwent abrupt swelling, giving rise to fine component microfibrils which showed varying degrees of organisation and alignment with the unaffected length of the fibril (Fig. 9).

Fibrils of all diameters throughout the scleral stroma appeared similarly susceptible to swelling and dissociation. At some sites fibres containing many swollen fibrils ran alongside zones of afibrillar, flocculent material, presumably the remains of dispersed collagen fibrils (Fig. 8). Atypical fibrils with broad diffuse crossbanding were common in association with total collagen fibril destruction (Fig. 5).

Fibroblasts were seen adherent to the surfaces of fibres containing swollen fibrils and also within extracellular lacunae where fibril loss had taken place (Fig. 10). Some of these cells appeared to be metabolically active, as shown by numerous profiles of dilated cisternae of rough-surfaced endoplasmic reticulum and large Golgi complexes within their cytoplasm. In contrast, others displayed degenerative changes including cell and nuclear membrane disruption and loss of organelles. Intact fibres with no 


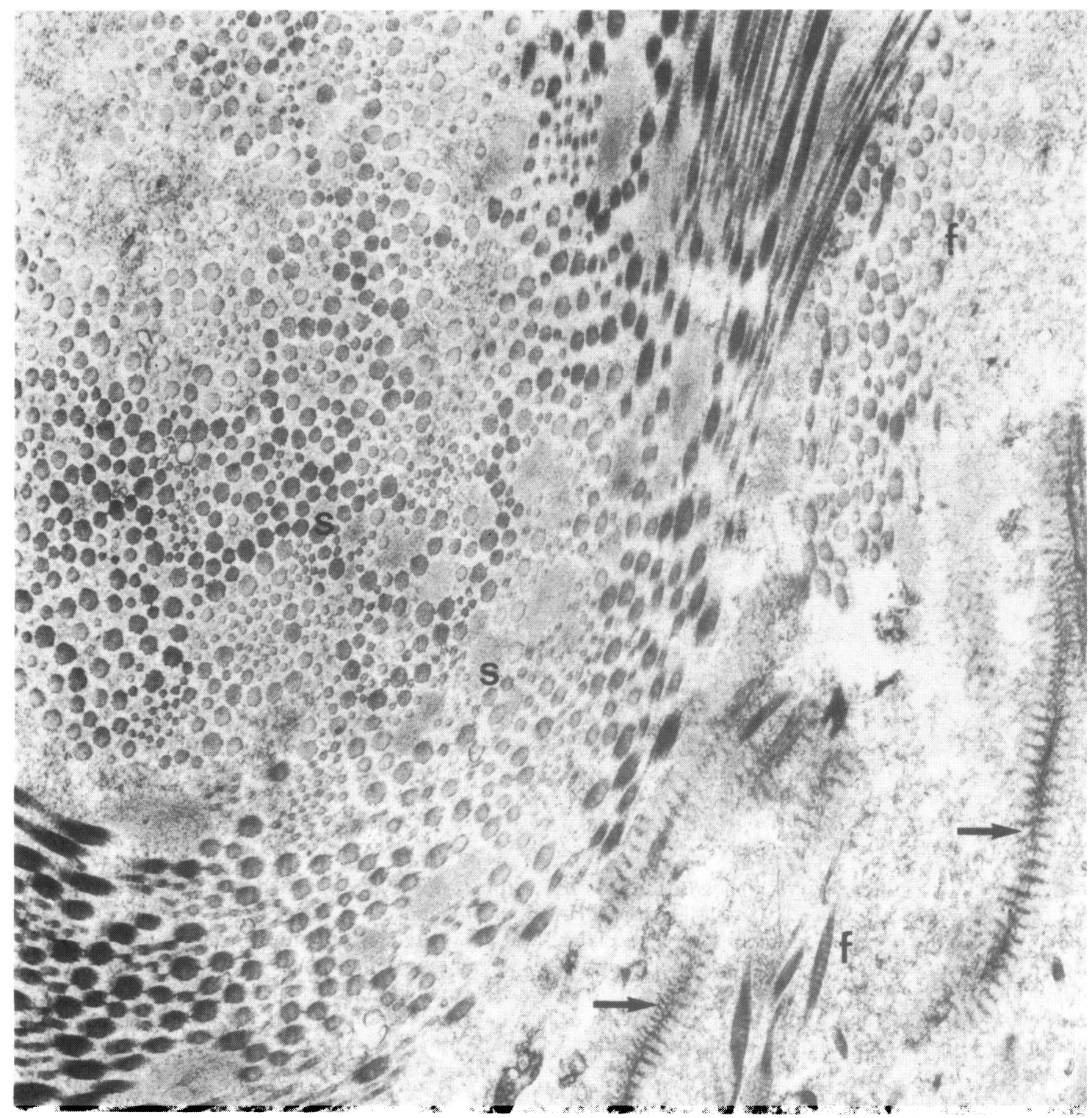

Fig. 5 Electron micrograph from an area of scleral stroma apparently intact by light microscopy shows variable swelling (s) and dissociation of collagen fibrils within one fibre. Few intact fibrils (f) remain in an adjacent fibre, where atypical crossbanded structures are present (arrows). $(\times 21000)$.

discernible fibril changes were also seen adjacent to regions where fibril loss was complete (Fig. 10).

\section{Discussion}

Electron microscopy of tissue from patients with necrotising scleritis shows clearly that the sclera is host to both extracellular and intracellular mechanisms of collagen degradation.
The interaction of phagocytic cells with collagen fibrils as described in this study has been recognised both at sites of rapid connective tissue turnover during morphogenesis in normal tissues, such as involuting uterus, periodontal ligament, and regressing hair follicles, ${ }^{5-7}$ and in pathological tissue destruction, as in rheumatoid synovial pannus. ${ }^{8}$ It has also been observed during the remodelling of scar tissue in dermal wounds." Both macrophages and fibroblasts have 


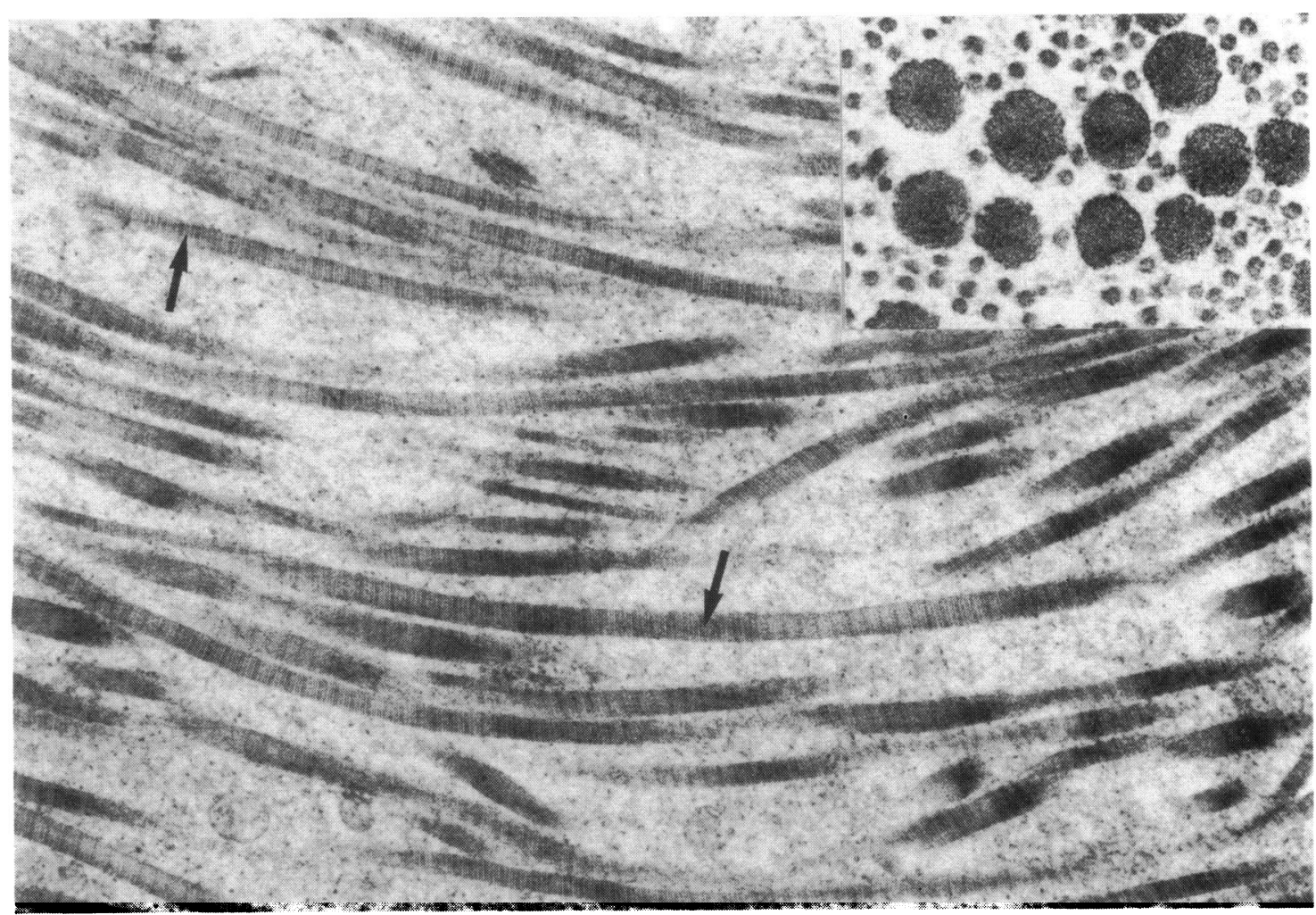

Fig. 6 Electron micrograph from deep sclera in case 7 showing early stages in fibril damage as diagonal or spiral clefts along the fibril length (arrows). $(\times 37800)$. Inset: In transverse section of outer sclera unaffected fibrils appear smooth contoured in comparison with the serrated profiles of swollen neighbours. Both coarse and fine fibrils are affected. $(\times 46000)$.

been reported to interact with collagen in this way.

It is generally accepted that, following their engulfment into deep invaginations of the cell or completely into cytoplasmic vacuoles, collagen fibres may be subjected to a microenvironment with sufficiently acid $\mathrm{pH}$ for lysosomal proteases to be active. ${ }^{10}$ However, neither the mechanisms of cell attachment to collagen nor the stimulus for phagocytosis are fully understood.

The extracellular disintegration of collagen fibrils represents an extremely dramatic and widespread degenerative change in the scleral stroma of these patients, which appears to be independent of close association between cells and the collagen fibrils. Extracellular destruction of collagen in corneal alkali burns and in the corneas of vitamin-A-deficient rats $^{11} 12$ has previously been attributed to the release of neutral proteinases, including collagenase, into the matrix. It seems likely that collagen breakdown by this pathway is the result of the action of a series of proteases operating in concert. ${ }^{13}$ Extracellular collagen degradation in the sclera takes place in the absence of infiltrating granulocytes. The native stromal fibrocytes which show a variety of morphological changes in necrotising scleritis, including activation and degeneration, are probably the major source of proteolytic enzymes in this situation.

Alternatively, the synchronous degradation of collagen fibrils throughout the stroma suggests that collagenolytic enzymes, perhaps located on fibrils in the matrix, may be undergoing simultaneous activation. ${ }^{14}$ The presence in the sclera of intact fibres adjacent to those showing total collagenolysis may indicate compartmentalisation of the connective tissue such that individual or small groups of fibroblasts may regulate biosynthesis of matrix macromolecules in discrete domains.

It is likely that extracellular collagenolysis in which fibrils unravel, lose their structure, and disperse into a flocculent solution corresponds to fibrinoid degeneration or hyalinisation of collagen as described in early histological studies of necrotising scleritis. ${ }^{24}$ Fibrin deposition was not found in conjunction with collagen degradation, though its presence was previously noted in particular around capillaries and at sites of cellular necrosis in the granuloma. ${ }^{\text {is }}$ 


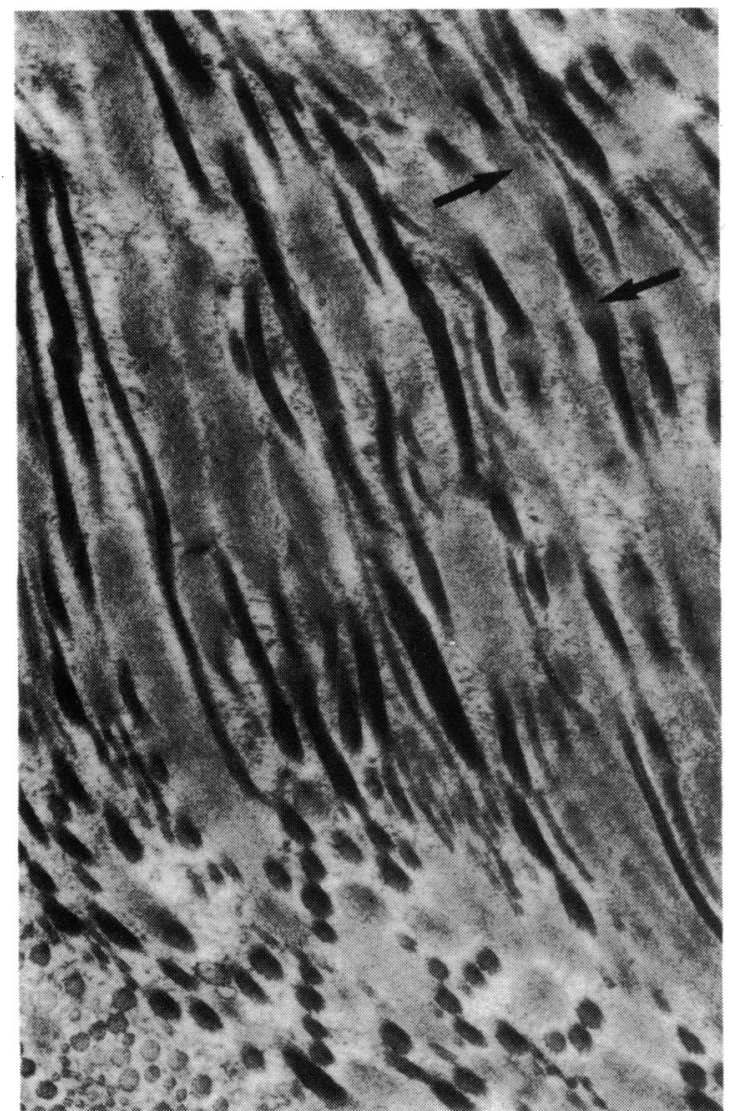

Fig. 7 Electron micrograph of scleral stroma in case 7 showing focal swelling along collagen fibrils in longitudinal section (arrows). $(\times 16500)$.

A number of experimental studies have shown swelling and unravelling of collagen fibrils in the cornea in response to immune complex deposition in the stroma. ${ }^{16-18}$ Mohos and Wagner were able to visualise immune complexes precipitated around collagen fibrils in the rabbit cornea by the use of ferritin-labelled antigens. ${ }^{17}$ They suggest that collagen fibril damage occurs during the phagocytic removal of immune complexes by granulocytes, and that fibrinoid is a mixture of immune precipitate and damaged collagen fibrils, although they failed to illustrate this clearly. Experimental studies have since confirmed that, at least in vitro, collagen can bind immune complexes, possibly due to the extensive chemical and structural similarity between the collagen molecule and subcomponent $\mathrm{Clq}$ of the first complement component. By competition for binding sites immune complexes may inhibit collagen-induced platelet aggregation, ${ }^{19}$ and thus by this mechanism potentiate inflammation.

The swelling of collagen fibrils produced experi-

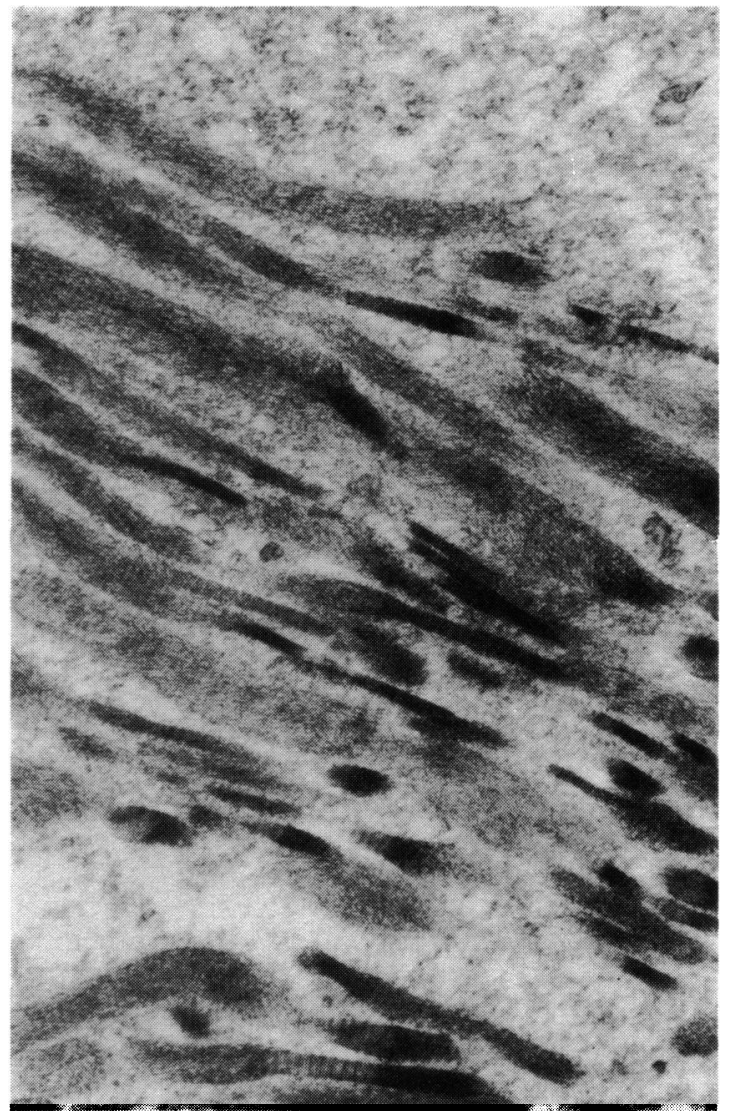

Fig. 8 Advanced collagen fibril swelling in one fibre and total loss of fibril structure in an adjacent area (top of field) in the scleral stroma of case 7. A diffuse periodicity of $31 \mathrm{~nm}$ is visible along damaged lengths of fibrils in comparison with $45 \mathrm{~nm}$ banding along intact regions. $(\times 19800)$.

mentally by immune precipitate deposition in the rabbit cornea ${ }^{17}$ was not as marked as that seen in the scleras of these patients. Moreover, there were no associated inflammatory cells present. No structures resembling immune aggregates were detected in conjunction with fibril damage in scleral tissue examined here. However, while it has been postulated that scleral necrosis results from immune complex deposition in association with the scleral vasculature, ${ }^{20}$ no specific labelling for complexes is possible because the putative antigens are unknown.

Both extracellular and intracellular degradation of dermal collagen have recently been described during methylcholanthrene-induced carcinogenesis in mice. ${ }^{21}$ When the cells of the epidermis became invasive, widespread collagen resorption involving the replacement of fibrils by amorphous or fine filamentous material, occurred extracellularly in the presence of activated fibroblasts. Phagocytosis of 


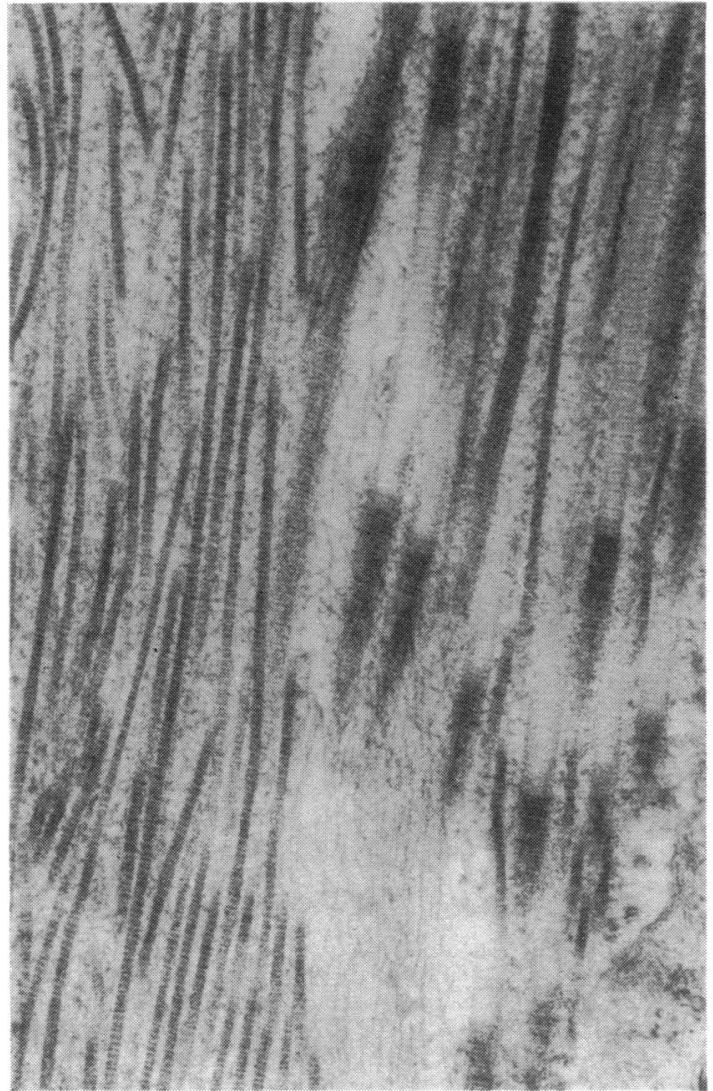

Fig. 9 Electron micrograph from scleral stroma in case 2 illustrates abrupt disintegration of collagen fibrils to reveal sprays of fine component microfibrils in longitudinal section. $(\times 36000)$.

collagen by fibroblasts or macrophages was seen in an early preinvasive phase and was resumed when advanced extracellular collagenolysis was evident. Although extracellular collagen destruction seems to be an early event in scleral damage during necrotising scleritis, detectable prior to granuloma formation in the tissue, no conclusions can be made concerning a possible mechanistic sequence of collagenolysis in this disease. It seems likely that both mechanisms may proceed simultaneously at different locations with respect to the granuloma.

The authors gratefully acknowledge the support by a grant from the Wellcome Trust.

\section{References}

1 Woessner JF Jnr. In: Gould BS, ed. Treatise on collagen. New York: Academic Press, 1968: 2B: 253-330.

2 Ashton N, Hobbs HE. Effect of cortisone on rheumatoid nodules of the sclera (scleromalacia perforans). Br J Ophthalmol 1952; 36: 373-84.

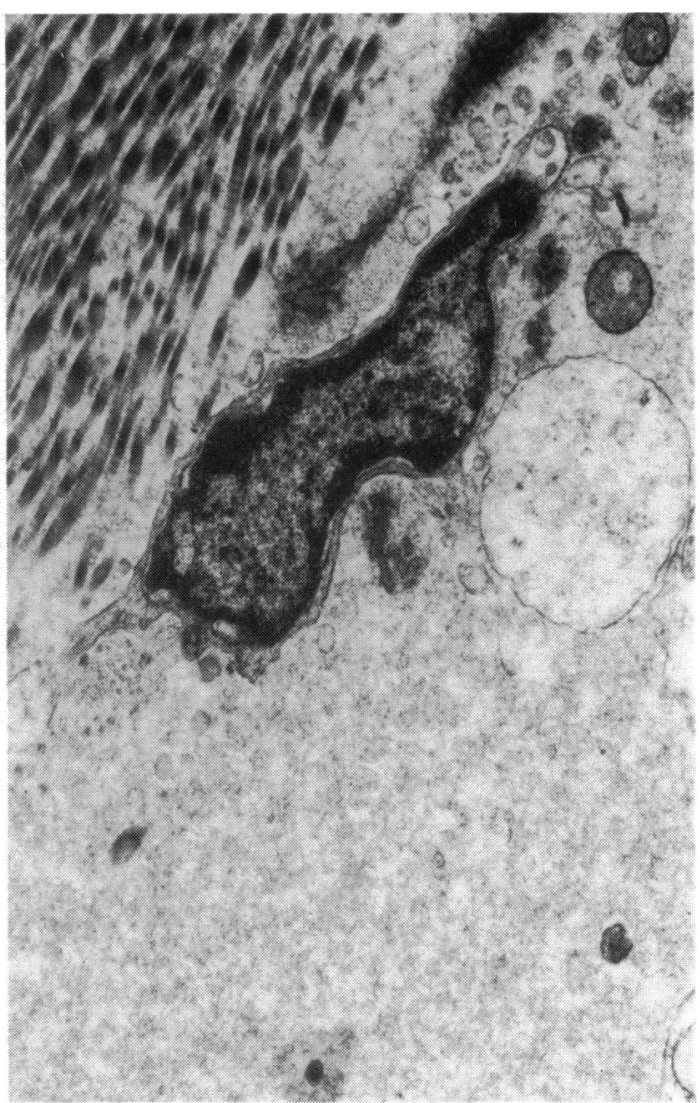

Fig. 10 Junction between adjacent fibres in scleral stroma of case 2 shows preservation of fibril structure in one, while few intact fibrils remain in the other. A scleral cell at the junction shows advanced degenerative changes. $(\times 16500)$.

3 Sevel D. Necrogranulomatous scleritis. Clinical and histological features. Am J Ophthalmol 1967; 64: 1125-34.

4 François J. Ocular manifestations in collagenoses. Adv Ophthalmol 1970; 23: 1-54.

5 Padykula HA. Cellular mechanisms involved in cyclic stromal renewal of the uterus. Anat Rec 1976; 184: 49-71.

6 Ten Cate AR, Syrbu S. A relationship between alkaline phosphatase activity and the phagocytosis and degradation of collagen by the fibroblast. $J$ Anat $1974 ; 117: 351-9$.

7 Parakkal PF. Role of macrophages in collagen resorption during the hair growth cycle. J Ultrastruct Res 1969; 29: 210-7.

8 Harris ED Jnr, Glauert AM, Murley AHG. Intracellular collagen fibres at the pannus-cartilage junction in rheumatoid arthritis Arthritis Rheum 1977; 20: 657-65.

9 McGaw WT, Ten Cate AR. A role for collagen phagocytosis by fibroblasts in scar remodelling: an ultrastructural stereologic study. J Invest Dermatol 1983; 81: 375-8.

10 Sellers A, Murphy G. Collagenolytic enzymes and their naturallyoccurring inhibitors. In: Hall DA, Jackson DS, eds. International review of connective tissue research. New York: Academic Press, 1981: 9: 151-90.

11 Berman M, Dohlman CH, Davison PF, Gnadinger M. Characterisation of collagenolytic activity in the ulcerating cornea. Exp Eye Res 1971; 11: 255-7. 
12 Pirie A, Werb Z, Burleigh MC. Collagenase and other proteinases in the cornea of the retinol-deficient rat. BrJ Nutr 1975; 34: 297-309.

13 Weiss JB, Sedowofia K, Jones C. Collagen degradation: a defended, multi-enzyme system. In: Viidik A, Vinist J, eds. Biology of collagen. New York: Academic Press, 1980: 11334.

14 Pardo A, Perez-Tamayo R. The presence of collagenase in collagen preparations. Biochim Biophys Acta 1975; 392: 12130.

15 Young RD, Watson PG. Microscopical studies of necrotising scleritis. I. Cellular aspects. Br J Ophthalmol 1984; 68: 770-780.

16 Germuth FG, Maumenee AE, Senterfitt LB, Pollack AD. Immuno-histologic studies on antigen-antibody reactions in the avascular cornea. J Exp Med 1962; 115: 919-28.
17 Mohos SC, Wagner BM. Damage to collagen in corneal immune injury. Arch Pathol 1969; 88: 3-20.

18 Young RD, Hembry RM, Watson PG, Dingle JT. Ultrastructural studies of corneal collagen during the development of experimental scleritis in rabbits. Diag Histopathol 1981; 4: 199-207.

19 Csako G, Suba EA, Herp A. On the reactivity of corneal collagen and subcomponent $\mathrm{Clq}$ of the complement system with human platelets and IgG-coated latex particles. Exp Eye Res 1983; 36: 403-14.

20 Watson PG. The nature and the treatment of scleral inflammation. Trans Ophthalmol Soc UK 1982; 102: 257-81.

21 Van den Hoof A, Tigchelaar-Gutter W. Stromal changes in experimental carcinogenesis in the skin. 1. Ultrastructural aspects of collagen degradation. Proc K Ned Akad Wetenschappen 1983; C86: 179-90. 